\title{
Comparison of Oxidative Stability of Monogalactosyl Diacylglycerol, Digalactosyl Diacylglycerol, and Triacylglycerol Containing Polyunsaturated Fatty Acids
}

\author{
Noor Yuslida Binti Hazahari, Masashi Hosokawa, Kazuo Miyashita* \\ Faculty of Fisheries Sciences, Hokkaido University, Hakodate, Japan \\ Email: *kmiya@fish.hokudai.ac.jp
}

How to cite this paper: Hazahari, N.Y.B., Hosokawa, M. and Miyashita, K. (2018) Comparison of Oxidative Stability of Monogalactosyl Diacylglycerol, Digalactosyl Diacylglycerol, and Triacylglycerol Containing Polyunsaturated Fatty Acids. Food and Nutrition Sciences, 9, 221-234.

https://doi.org/10.4236/fns.2018.93017

Received: November 1, 2017

Accepted: March 12, 2018

Published: March 15, 2018

Copyright $\odot 2018$ by authors and Scientific Research Publishing Inc. This work is licensed under the Creative Commons Attribution International License (CC BY 4.0).

http://creativecommons.org/licenses/by/4.0/

\begin{abstract}
Oxidative stability of three different lipid classes, namely, monogalactosyl diacylglycerol (MGDG) and digalactosyl diacylglycerol (DGDG) from spinach and edible brown seaweed (Akamoku) and triacylglycerol (TAG) of linseed oil was compared. Analysis of oxygen consumption and polyunsaturated fatty acid (PUFA) composition demonstrated that spinach DGDG had the highest oxidative stability, followed by Akamoku DGDG, Akamoku MGDG, spinach MGDG, and linseed TAG. These results disagree with the order of oxidative stability expected from the average number of bis-allylic positions of each lipid. Additionally, DGDG constituents of both spinach and Akamoku showed higher oxidative stability than their MGDG constituents. The unusual oxidative stability of MGDG and DGDG could be conferred by the protection of bis-allylic positions of the PUFA against oxidative attack by the galactosyl moiety of the GL.
\end{abstract}

\section{Keywords}

Oxidative Stability, Glyceroglycolipids, Monogalactosyl Diacylglycerol, Digalactosyl Diacylglycerol, Triacylglycerol, Polyunsaturated Fatty Acids

\section{Introduction}

Monogalactosyl diacylglycerol (MGDG), digalactosyl diacylglycerol (DGDG), and sulfoquinovosyl diacylglycerol (SQDG) are the main lipid constituents of plant leaves (Figure 1) [1]. In addition, these polar lipids are known to be the major constituents of seaweeds [2] [3]. Together, MGDG and DGDG account 


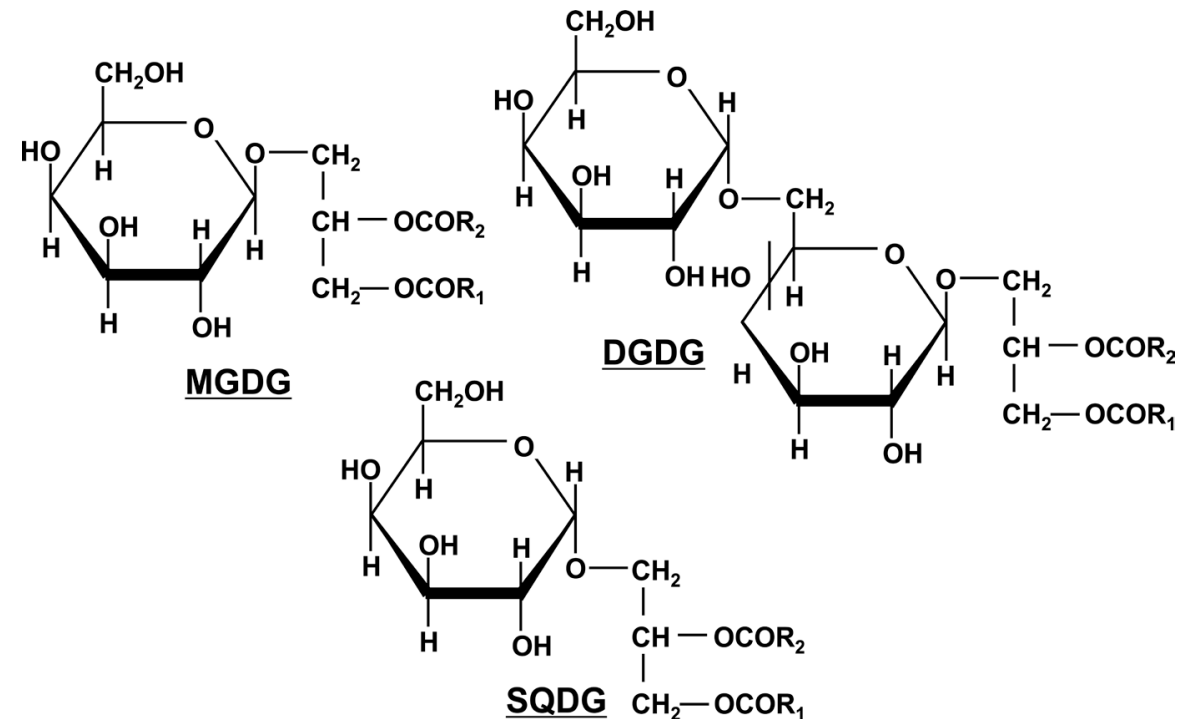

Figure 1. Structures of MGDG, DGDG, and SQDG. MGDG, monogalactosyl diacylglycerol; DGDG, digalactosyl diacylglycerol; SQDG, sulfoquinovosyl diacylglycerol.

for more than half of the total membrane lipids in plant leaves [4]. Therefore, these glyceroglycolipids (GLs) are thought to play important roles in the photosynthetic membranes of higher plants, algae, and bacteria [5].

GLs in higher plants have also been characterized as having a uniquely high content of polyunsaturated fatty acids (PUFAs), particularly $\alpha$-linolenic acid (LN, 18:3n-3) [4]. In contrast, most PUFAs in seaweed GL are stearidonic acid (SA, 18:4n-3), arachidonic acid (AA, 20:4n-6), and eicosapentanoic acid (EPA, 20:5n-3) [2] [6]. All of these PUFAs are more easily oxidized than the more stable unsaturated fatty acids such as oleic acid (OA, 18:1n-9) and linoleic acid (LA, 18:2n-6) [7] [8] [9]. Despite the higher levels of oxidatively unstable PUFAs, GLs, a main lipid component of the chloroplast, are continuously subjected to oxidative stress because of the absorption of light energy during photosynthesis.

It is generally accepted that autoxidation of PUFA proceeds in a free radical chain reaction through three stages: i) initiation, ii) propagation, and iii) termination. The free radical theory of autoxidation was defined as the attack of oxygen at the allylic position leading to the formation of unsaturated hydroperoxides. These hydroperoxides can then decompose into peroxyl and alkoxyl radicals, which followed by secondary oxidation products including aldehydes, ketones, alcohols, and acids. Impaired taste, flavor, and texture of foods are among the adverse effects of secondary oxidation products, which may be toxic compounds. Thus, lipid oxidation is a major concern that can shorten the shelf life of foods.

Plants possess intrinsic antioxidant compounds for defense against oxidation, including ascorbate, glutathione, phenolic compounds, tocopherols, and carotenoids. Innate enzymes such as superoxide dismutase, catalase, ascorbate peroxidase, and glutathione reductase are capable of scavenging reactive oxygen species and eventually protecting plants from oxidative stress [10] [11] [12]. 
Another defense system against oxidative stress in photosynthetic tissues may be related to the presence of PUFA in the form of GL. However, few studies have examined the resistance of GL to oxidation [4]. Therefore, it is very important to determine the role of PUFA in the form of GL and its characteristics response. This study evaluated the oxidative stability of GL by comparing the oxidative stability of 5 types of lipids: spinach MGDG, spinach DGDG, brown seaweed MGDG, brown seaweed DGDG, and linseed oil triacylglycerol (TAG).

\section{Experimental Procedures}

\subsection{Sample}

Spinach powder (GABAN Co. Ltd., Tokyo, Japan) was obtained from a local food market in Hakodate, Japan. Edible brown seaweed, Sargassum horneri (Turner) (common name: Akamoku), was collected from Hakodate, Hokkaido, Japan, and the powder was prepared by Cokey Co., Ltd. (Tokyo, Japan). Linseed oil was purchased from Wako Pure Chemical Ltd. (Osaka, Japan).

\subsection{Referenced Compound and Reagent}

Silica gel (BW-60F) for column chromatography was purchased from Fuji Sylysia Chem. Ltd. (Kasugai, Aichi, Japan). Activated Carbon and Celite (545 RVS) were purchased from Nacalai Tesque, Inc. (Kyoto, Japan). MGDG, DGDG, and SQDG standards were purchased from Lipid Products (Redhill, UK), while triolein and tricaprylin as medium chain TAG (MCT) were obtained from Wako Pure Chemical. All other chemicals and solvents used in this study were of analytical grade and high-performance liquid chromatography (HPLC)-grade solvents were used for HPLC analysis.

\subsection{Sample Preparation}

Spinach powder $(c a .2 \mathrm{~kg}$ ) was soaked in methanol $(12,000 \mathrm{~mL})$ at room temperature, which was incubated in the dark overnight (approximately $16 \mathrm{~h}$ ) [13]. The extract was filtered through a ceramic filter funnel lined with filter paper (No. 2, Qualitative Filter Paper; 150 mm; Advantec ${ }^{\oplus}$; Tokyo, Japan). The filtrates were pooled and the solvent was removed using a pilot scale rotary evaporator at less than $40^{\circ} \mathrm{C}$. Traces of solvent remaining in the extract were completely removed in the dark under vacuum, leaving a dark green viscous liquid. This dark green viscous liquid was collected by dissolution in an equivalent volume of methanol, which was designated as crude spinach lipids.

Crude spinach lipids were further dissolved in chloroform-methanol-water $(10: 5: 3, \mathrm{v} / \mathrm{v} / \mathrm{v})$ [13]. The solution was placed into a separatory funnel for liquid-liquid distribution. After shaking, the funnel was allowed to stand overnight. The lower layer, a mixed lipid layer with methanol and chloroform, was collected and again dissolved in water using a new separatory funnel. In this case, the same volume of water as that in the first separation, was added; after shaking, the funnel was allowed to stand for overnight separation. The lower layer 
was then concentrated under a vacuum in a rotary evaporator. Remaining traces of organic solvents and water were removed in a desiccator (approximately 3 days) under a high vacuum, leaving the sample in an amber-colored rotary flask. Total lipids of spinach (ca. $20 \mathrm{~g}$ ) were eventually collected by dissolving in an equivalent volume of chloroform and subjected to consequent analysis or stored at $-30^{\circ} \mathrm{C}$ in an equivalent volume of ethanol. The same procedure was used for Akamoku powder.

\subsection{Lipid Class Analysis}

Total lipids of spinach and Akamoku were subjected to lipid class analysis by preparative thin layer chromatography (TLC) [14]. The lipid fraction was dissolved in chloroform-methanol-water $(65: 25: 4, \mathrm{v} / \mathrm{v} / \mathrm{v})$ and spotted onto a $0.25-\mathrm{mm}$ silica gel plate (Silica gel 60G; Merck, Darmstadt, Germany). The plate was developed with the same cocktail mix solvents of chloroform-methanol-water $(65: 25: 4, \mathrm{v} / \mathrm{v} / \mathrm{v})$ and the spots were visualized by spraying the plate with orcinol-sulfuric acid or Dittmer reagent, followed by charring. The lipid sample was also analyzed by preparative TLC with the same specification of silica gel plate mentioned previously but using $n$-hexane-diethyl ether-acetic acid (80:20:1, v/v/v) as the developing solvent. The spots were detected using $60 \%$ aqueous sulfuric acid charring.

The chromatogram was photographed with a digital camera and the image of the silica gel plate was acquired and transferred to a computer. The image was cropped and saved in bitmap format. The percentage ratio of each lipid fraction (as compared to standards) in the sample was expressed as the bitmap percentage of the total bitmap intensities [14].

\subsection{Purification of GL from Spinach and Akamoku}

Total lipids of spinach and Aakamoku (ca. $20 \mathrm{~g}$ ) were first passed through a column $(70 \times 6 \mathrm{~cm}$ i.d.) packed with a chloroform slurry mixture of silica gel. The whole column was wrapped with aluminum foil to protect the GL from light-induced degradation. The elution was first conducted with chloroform (approximately $3000 \mathrm{~mL}$ ) and then with acetone (approximately 13,000 mL). Fractions eluted with acetone were used as GL (through continuous elution, with appropriately adjusting the flow rate manually, for more than a week). The first whole dark green-black fraction was designated as MGDG and the consequent fractions (approximately 24 fractions) of each $500 \mathrm{~mL}$ collected with acetone (clear light green solution) were designated as the DGDG.

Although the absence of chlorophyll was confirmed in the DGDG, a trace amount of chlorophyll was detected in the MGDG on TLC. Therefore, MGDG (ca. $4 \mathrm{~g})$ was further refined using a carbon column $(70 \times 6 \mathrm{~cm}$ i.d.) packed with $90 \%$ of ethanol in chloroform slurry of activated carbon (ca. $250 \mathrm{~g}$ ). Carbon was first heated at $110^{\circ} \mathrm{C}$ for $1.5 \mathrm{~h}$ prior to mixing with $90 \%$ of ethanol in chloroform. Elution was conducted with gradually decreasing concentrations of etha- 
nol; $90 \%$ ethanol in chloroform (approximately $1000 \mathrm{~mL}$ ), 50\% ethanol in chloroform (approximately $1500 \mathrm{~mL}$ ), and finally 100\% chloroform (approximately $10,000 \mathrm{~mL}$ ). The fractions eluted with $50 \%$ ethanol in chloroform and $100 \%$ chloroform were used as the MGDG. Purified MGDG and DGDG were used as Spinach MGDG, Spinach DGDG, Akamoku MGDG, and Akamoku DGDG.

\subsection{Purification of TAG from Linseed Oil}

Linseed oil was separated by two types of column chromatography [15]. To remove tocopherols and pigments, linseed oil (ca. $25 \mathrm{~g}$ ) was first passed through a column $(50 \times 4 \mathrm{~cm}$ i.d.) packed with an $n$-hexane slurry mixture of activated carbon $(100 \mathrm{~g})$ and Celite $(100 \mathrm{~g})$. The carbon was first heated at $110^{\circ} \mathrm{C}$ for $1.5 \mathrm{~h}$ prior to mixing with Celite and $n$-hexane. Elution was performed with $n$-hexane (1200 mL).

The obtained oil (ca.10 g) was again refined using a silicic acid column $(50 \times$ $4 \mathrm{~cm}$ i.d.) packed with an $n$-hexane slurry of silica gel (200 g). Elution was conducted with $n$-hexane $(200 \mathrm{~mL}), n$-hexane-diethyl ether $(98: 2, \mathrm{v} / \mathrm{v})(200 \mathrm{~mL})$, and $n$-hexane-diethyl ether $(90: 10, \mathrm{v} / \mathrm{v})(1200 \mathrm{~mL})$. The final fraction eluted with $n$-hexane-diethyl ether (90:10) was used as Linseed TAG.

To confirm the absence of impurities, the TAG fraction was subjected to preparative TLC. The lipid fraction was spotted onto a $0.25-\mathrm{mm}$ silica gel plate. The plate was again developed with $n$-hexane-diethyl ether (60:40, v/v) and spots were detected with iodine vapor or $60 \%$ aqueous sulfuric acid charring as compared to standard triolein.

\subsection{Tocopherol Analysis of Spinach GL, Akamoku GL, and Linseed TAG}

Tocopherol analysis was performed for all 5 types of purified lipids, Spinach MGDG and DGDG, Akamoku MGDG and DGDG, and Linseed TAG, with a Hitachi HPLC system equipped with a pump (Hitachi L-2130, Hitachi Seisakusho, Co., Tokyo, Japan) and fluorescence detector (Hitachi L-2485) [4]. Analysis was conducted on a silica column (Si 60, $250 \times 4.6 \mathrm{~mm}$ i.d.; Kanto Chemical Co., Tokyo, Japan) protected with a guard column $(15 \times 3.2 \mathrm{~mm})$ with the same stationary phase. The mobile phase was $n$-hexane-2-propanol $(99.2: 0.8, \mathrm{v} / \mathrm{v})$ at a flow rate of $1.0 \mathrm{~mL} / \mathrm{min}$. The fluorescence detector was set at Ex. $298 \mathrm{~nm}$ and Em. $325 \mathrm{~nm}$.

\subsection{Fatty Acid Composition of Spinach GLs, Akamoku GLs, and Linseed TAG}

The fatty acid composition of all 5 purified lipids was determined by gas chromatography (GC) after conversion of fatty acyl groups in the lipid to their methyl esters as described by Prevot and Modret [16], with slight modifications. Briefly, to an aliquot of total lipid (ca. $20 \mathrm{mg}$ for GLs and $10 \mathrm{mg}$ for TAG), $1 \mathrm{~mL}$ of $n$-hexane and $0.2 \mathrm{~mL}$ of $2 \mathrm{~N} \mathrm{NaOH}$ in methanol solution were added, vortexed for $10 \mathrm{~s}$, and incubated at $50^{\circ} \mathrm{C}$ for $30 \mathrm{~s}$. Next, $0.2 \mathrm{~mL}$ of $2 \mathrm{~N} \mathrm{HCL}$ in methanol 
was added to the solution followed by vortexing for $1 \mathrm{~min}$. The mixture was separated by centrifugation at $1000 \times g$ for $5 \mathrm{~min}$. The upper hexane layer containing fatty acid methyl esters was recovered and subjected to GC. GC analysis was performed using Shimadzu GC-2014 (Shimadzu Corporation, Kyoto, Japan) equipped with a flame-ionization detector and capillary column (Supelco ${ }^{\mathrm{Tm}}$ Column; Omegawax-320; $30 \mathrm{~m} \times 0.32 \mathrm{~mm}$ i.d.; Sigma-Aldrich, St. Louis, MO, USA). The detector, injector, and column temperatures were $260^{\circ} \mathrm{C}, 250^{\circ} \mathrm{C}$, and $200^{\circ} \mathrm{C}$, respectively. The carrier gas was helium at a flow rate of $50 \mathrm{kPa}$. The fatty acid content was expressed as a weight percentage of total fatty acids.

\subsection{Oxidation Analysis of Purified Lipids}

Each purified lipid $(100 \mathrm{mg}$ ) was placed in a $2-\mathrm{mL}$ aluminum sealed vial with a butyl gum septum (GL Science; Tokyo, Japan) and then incubated at $50^{\circ} \mathrm{C}$ in the dark. The level of oxygen and nitrogen in the headspace gas of the vial was estimated using a GC system (Shimadzu GC-14B) equipped with a thermal conductivity detector and stainless steel column $(3 \mathrm{~m} \times 3.0 \mathrm{~mm}$ i.d. $)$ packed with a molecular sieve 5A (GL Science) as described by Cho et al. [9]. The temperatures at the injection port, detector port, and column oven were $120^{\circ} \mathrm{C}, 120^{\circ} \mathrm{C}$, and $70^{\circ} \mathrm{C}$, respectively. The helium flow was $50 \mathrm{kPa}$. Three separate vials containing similar samples were prepared and incubated. A small portion $(20 \mu \mathrm{L})$ of the headspace gas was taken from each vial using a microsyringe through the butyl gum septum at selected times during oxidation. Before the incubation, the ratio of peak area of oxygen to nitrogen was around 0.26 . The ratio decreased with the progress of lipid oxidation. The percentage decrease (\%) in oxygen could be calculated from the changes in the oxygen to nitrogen ratio compared to the ratio before incubation. In Three replicate measurements of all data and value at different oxidation times of the stored samples were expressed as the mean $\pm \mathrm{SD}$ ( $n$ $=3$ ).

After oxidation, PUFA contents were again analyzed by GC. The preparation of fatty acid methyl esters and GC analysis was performed as described above.

\section{Results}

\subsection{Lipid Class Analysis}

When each lipid composition of both Spinach and Akamoku GLs was visually analyzed based on the spot intensities of TLC (Figure 2), the overall distribution of their main lipid classes was determined and is shown in Table 1. Only MGDG and DGDG from both Spinach and Akamoku were analyzed, while SQDG was not analyzed because it generally showed low levels in Spinach and thus was not the focus of this research.

\subsection{Purification of GL and Linseed TAG}

Purified MGDG and DGDG from Spinach and Akamoku showed spots corresponding to MGDG and DGDG standards on TLC, respectively. In addition, 


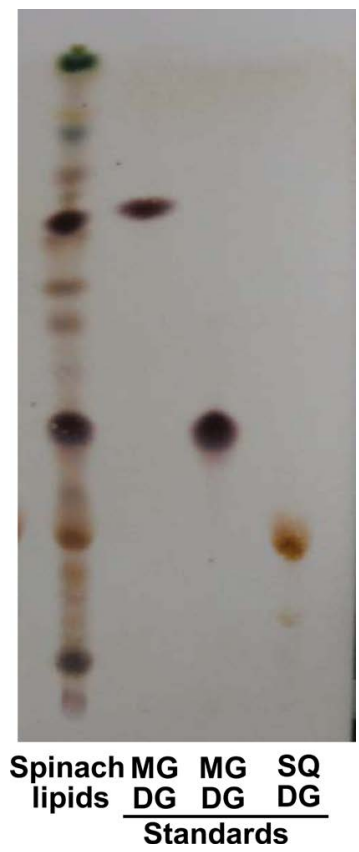

Figure 2. Representative TLC of spinach lipids. Sample solution was spotted on a $0.25-\mathrm{mm}$ silica gel plate. The plate was developed with chloroform-methanol-water $(65: 25: 4, \mathrm{v} / \mathrm{v} / \mathrm{v})$ and the spots were visualized by charring.

Table 1. Lipid class composition of Spinach GL and Akamoku GL.

\begin{tabular}{ccc}
\hline GL class (\% of total GL) & Spinach & Akamoku \\
\hline MGDG & 50 & 62 \\
DGDG & 43 & 8 \\
SQDG & 7 & 30
\end{tabular}

visible spots with small streaks of chlorophyll were observed during TLC analysis with Spinach MGDG and Akamoku MGDG, while both Spinach DGDG and Akamoku DGDG were free from spots containing small streaks of chlorophyll after the first purification. However, after both MGDGs were subjected to a second purification with a carbon column, only a single spot corresponding to MGDG standard was detected on the TLC for each type of sample.

Purified Linseed TAG, in contrast, showed only a single spot corresponding to the lipid standard triolein on analytical TLC. No other impurities such as free fatty acids, monoacylglycerol, or diacylglycerol were detected.

\subsection{Tocopherol Analysis}

HPLC analysis either showed complete removal of tocopherols for nearly all types of lipids or only a very small amount of this antioxidant compound remained for the remaining few types of lipids. Complete removal was confirmed after double purification of all GL samples. Minute and negligible amounts of tocopherol, however, were detected for samples of Linseed TAG, as reported by Shimajiri et al. [17], which do not affect comparisons of the oxida- 
tive stability results.

\subsection{Fatty Acid Composition}

The overall fatty acid composition of purified GLs and Linseed TAG before and after oxidation is shown in Table 2. The main fatty acid in Linseed TAG and both Spinach MGDG and DGDG before oxidation was LN (18:3n-3), which showed weight percentages of $43.47 \%, 48.96 \%$, and $77.53 \%$, respectively. Only Spinach GL contained 16:3n-3, which accounted for $11.56 \%$ and $2.91 \%$ for Spinach MGDG and DGDG in weight percentage. In contrast, Akamoku MGDG and DGDG contained palmitic acid (PA; 16:0) as their main fatty acids, making up $35.92 \%$ and $24.09 \%$ of the overall weight percentage. In addition, Akamoku GLs were abundant in OA (18:1n-9), AA (20:4n-6), SDA (18:4n-3), and EPA (20:5n-3).

Table 3 shows the number of bis-allylic positions per molecule of each lipid. The number of bis-allylic positions per molecule or gram of each lipid was determined from the molar concentration of each PUFA and the mean molecular

Table 2. Major fatty acid composition of oxidation substrates before and after the oxidation.

\begin{tabular}{|c|c|c|c|c|c|c|c|c|c|c|}
\hline & \multicolumn{2}{|c|}{ Spinach MGDG } & \multicolumn{2}{|c|}{ Spinach DGDG } & \multicolumn{2}{|c|}{ Akamoku MGDG } & \multicolumn{2}{|c|}{ Akamoku DGDG } & \multicolumn{2}{|c|}{ Linseed TAG } \\
\hline $\begin{array}{c}\text { Fatty } \\
\text { acid } \\
\text { (wt\%) }\end{array}$ & $\begin{array}{c}\text { Before } \\
\text { oxidation }\end{array}$ & $\begin{array}{c}\text { After } \\
\text { oxidation }\end{array}$ & $\begin{array}{c}\text { Before } \\
\text { oxidation }\end{array}$ & $\begin{array}{c}\text { After } \\
\text { oxidation }\end{array}$ & $\begin{array}{c}\text { Before } \\
\text { oxidation }\end{array}$ & $\begin{array}{c}\text { After } \\
\text { oxidation }\end{array}$ & $\begin{array}{c}\text { Before } \\
\text { oxidation }\end{array}$ & $\begin{array}{c}\text { After } \\
\text { oxidation }\end{array}$ & $\begin{array}{c}\text { Before } \\
\text { oxidation }\end{array}$ & $\begin{array}{c}\text { After } \\
\text { oxidation }\end{array}$ \\
\hline $14: 00$ & $0.28 \pm 0.00$ & $1.00 \pm 0.09$ & $0.06 \pm 0.00$ & $0.08 \pm 0.00$ & $10.23 \pm 0.21$ & $11.07 \pm 0.27$ & $5.56 \pm 0.10$ & $5.86 \pm 0.01$ & $0.04 \pm 0.01$ & $3.08 \pm 5.05$ \\
\hline $16: 01$ & $0.26 \pm 0.03$ & $0.84 \pm 0.05$ & $0.16 \pm 0.03$ & $0.17 \pm 0.02$ & $8.90 \pm 0.08$ & $9.06 \pm 0.14$ & $5.15 \pm 0.06$ & $5.36 \pm 0.01$ & $0.07 \pm 0.00$ & $0.16 \pm 0.06$ \\
\hline $16: 03$ & $11.56 \pm 0.22$ & $6.12 \pm 0.63$ & $2.91 \pm 0.07$ & $3.83 \pm 0.02$ & $\mathrm{ND}$ & $\mathrm{ND}$ & $\mathrm{ND}$ & $\mathrm{ND}$ & ND & ND \\
\hline $18: 00$ & $0.40 \pm 0.01$ & $1.26 \pm 0.10$ & $0.70 \pm 0.03$ & $0.76 \pm 0.01$ & $0.89 \pm 0.01$ & $0.93 \pm 0.01$ & $0.33 \pm 0.01$ & $0.36 \pm 0.01$ & $3.62 \pm 0.02$ & $11.55 \pm 2.46$ \\
\hline $18: 1 n-9$ & $1.47 \pm 0.07$ & $3.87 \pm 0.23$ & $0.86 \pm 0.04$ & $0.97 \pm 0.01$ & $14.80 \pm 0.13$ & $14.58 \pm 0.13$ & $7.26 \pm 0.04$ & $7.25 \pm 0.16$ & $26.18 \pm 0.06$ & $40.61 \pm 10.34$ \\
\hline $18: 3 n-3$ & $48.96 \pm 1.37$ & $26.08 \pm 2.31$ & $77.53 \pm 0.14$ & $75.28 \pm 0.12$ & $1.13 \pm 0.02$ & $1.02 \pm 0.02$ & $5.47 \pm 0.02$ & $5.35 \pm 0.00$ & $43.47 \pm 0.03$ & $1.75 \pm 1.75$ \\
\hline $18: 4 n-3$ & ND & ND & ND & ND & $0.67 \pm 0.01$ & $0.56 \pm 0.02$ & $11.29 \pm 0.05$ & $10.64 \pm 0.06$ & ND & ND \\
\hline $20: 00$ & $0.35 \pm 0.24$ & $0.45 \pm 0.05$ & $0.02 \pm 0.00$ & $0.02 \pm 0.00$ & $0.11 \pm 0.02$ & $0.15 \pm 0.01$ & $0.05 \pm 0.00$ & $0.07 \pm 0.00$ & $0.16 \pm 0.01$ & ND \\
\hline $20: 4 n-6$ & ND & $\mathrm{ND}$ & ND & $\mathrm{ND}$ & $3.04 \pm 0.06$ & $2.61 \pm 0.16$ & $12.15 \pm 0.11$ & $11.30 \pm 0.01$ & ND & ND \\
\hline $20: 5 n-3$ & ND & ND & ND & ND & $0.46 \pm 0.08$ & $0.42 \pm 0.03$ & $9.64 \pm 0.15$ & $9.20 \pm 0.45$ & ND & ND \\
\hline
\end{tabular}

ND: not detected; PUFA: polyunsaturated fatty acids; MUFA: monounsaturated fatty acids; SFA: saturated fatty acids.

Table 3. Average number of bis-allylic positions of substrate lipids.

\begin{tabular}{cccccc}
\hline & \multicolumn{3}{c}{ Spinach } & \multicolumn{2}{c}{ Akamoku } \\
\cline { 2 - 4 } Total number of bis-allylic positions & MGDG & DGDG & MGDG & DGDG \\
\cline { 2 - 4 } Per molecule Per g lipid $(\times 6.02 \times 1020)$ & 1.73 & 1.71 & 0.29 & 1.54 \\
& 2.18 & 1.8 & 0.37 & 1.62 \\
\hline
\end{tabular}


weight (MW) of each lipid. The molar concentration of PUFA was calculated based on the weight percentage of PUFA before oxidation (Table 2). The mean MW of each lipid was obtained from the mean MW of all fatty acyl moieties and MW of the galactosyl and glycerol moieties. MWs were as follows: Spinach MGDG, 796.03; Spinach DGDG, 949.03; Akamoku MGDG, 776.97; Akamoku DGDG, 950.38, and Linseed TAG, 875.16. Based on the MW of each lipid and molar concentrations of PUFAs, the highest average number of bis-allylic positions per molecule was observed for Spinach DGDH, followed by Spinach MGDG, Akamoku DGDG, Linseed TAG, and Akamoku MGDG (Table 3).

\subsection{Stability of Purified Lipids in Bulk Phase}

When the oxidative stability of different types of lipids was compared by measuring the decrease in oxygen concentration in the bulk phase (Figure 3), the stability was the highest for Spinach DGDG, followed by Akamoku DGDG and MGDG, and Spinach MGDG. Linseed TAG was shown to have the lowest oxidative stability. The oxidative stability of polyunsaturated lipids is decreased with an increasing number of bis-allylic positions [18]. Based on the number of bis-allylic positions of each lipid (Table 3), Spinach DGDG was expected to be easily oxidized as compared with Akamoku DGDG, Linseed TAG, and Akamoku MGDG. However, the oxidative stability results, shown in Figure 3, were quite different from the expected average number of bis-allylic positions. In addition, both GLs with the DGDG constituent were shown to be more stable than their MGDG constituent when they showed a mild decrease in oxygen concentration over time (Figure 3). Although Spinach and Akamoku MGDG were

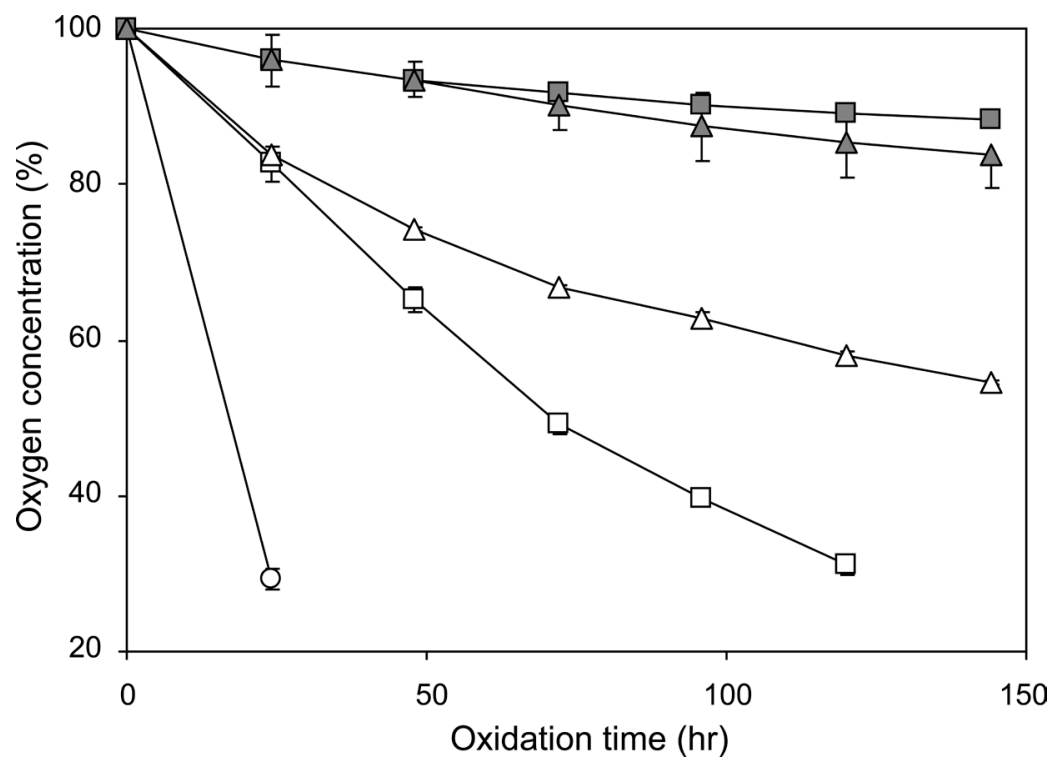

Figure 3. Oxidative stability of Spinach MGDG (open square), Spinach DGDG (closed square), Akamoku MGDG (open triangle), Akamoku DGDG (closed triangle), and Linseed TAG (open circle). Stability was analyzed by measuring the decrease in oxygen in the headspace gas of the vial. Oxidation was performed at $50^{\circ} \mathrm{C}$ in the dark. The data are expressed as the mean \pm SD of three independent experiments. 
more easily oxidized than the corresponding DGDG, they were still much more stable compared to Linseed TAG.

The characteristic oxidative stability of MGDG, DGDG, and TAG was also confirmed by analyzing the changes in the fatty acid composition of these substrates before and after oxidation (Table 2). Table 2 shows that LN was the major PUFA in Spinach MGDG, Spinach DGDG, and Linseed TAG. After oxidation, the amount of LN in Linseed TAG was greatly reduced to only $1.75 \%$ in weight percentage, showing a nearly $96 \%$ loss in the content ratio. In contrast, the LN content in Spinach MGDG was reduced by nearly half (a content loss of approximately $47 \%$ ), whereas its DGDG counterpart showed only a slight loss of approximately $3 \%$.

All samples showed a reduction in the total content of PUFA after oxidation. The differences were calculated from Table 2. Spinach MGDG showed a difference of $36.92 \%$, while Spinach DGDG showed a difference of only $4.96 \%$ in weight percentage. Akamoku MGDG exhibited a difference of $1.13 \%$ and Akamoku DGDG showed a minimal difference of $2.42 \%$ in weight percentage. Linseed TAG showed the greatest difference of $55.50 \%$ in weight percentage. In general, the total loss in content ratio with respect to the difference calculated in weight percentage was approximately 51\% (Spinach MGDG), 6\% (Spinach DGDG), 10\% (Akamoku MGDG), 5\% (Akamoku DGDG), and 92\% (Linseed TAG). The maximal loss in content ratio was observed for Linseed TAG, followed by Spinach MGDG, Akamoku MGDG, and then Spinach DGDG and finally Akamoku DGDG. Both DGDG from Spinach and Akamoku showed the lowest reduction in the content ratio as compared to their MGDGs constituent.

\section{Discussion}

PUFA oxidation generally proceeds through a free radical chain reaction [19] [20]. In this reaction, the rate-limiting step is the abstraction of hydrogen radical $(\mathrm{H} \bullet)$ from the substrate PUFA to form the PUFA free radical. Because this hydrogen abstraction occurs at the bis-allylic positions $\left(\mathrm{CH}=\mathrm{CH}-\mathrm{CH}_{2}-\mathrm{CH}=\mathrm{CH}\right)$ present in PUFA and the susceptibility of PUFA to oxidation depends on the availability of bis-allylic hydrogens, the oxidative stability of each PUFA is inversely proportional to the number of bis-allylic positions in the molecule. Therefore, among the relative oxidative stabilities of major PUFAs contained in Spinach GLs, Akamoku GLs, and Linseed TAG, EPA (20:5n-3) is most rapidly oxidized, followed by SDA (18:4n-3), AA (20:4n-6), 16:3n-3, LN (18:3n-3), and LA (18:2n-6) [7] [9] [21].

GL from marine lipid sources, both Akamoku MGDG and DGDG, showed high percentages of EPA. Particularly, Akamoku DGDG showed a higher percentage of EPA with a higher mean number of bis-allylic positions per molecule than that of Linseed TAG. Upon comparing the difference in the mean number of bis-allylic positions per molecule and the level of EPA, the oxidative stability of Akamoku DGDG was expected to be lower than that of Linseed TAG and 
Akamoku MGDG. However, the stability of Akamoku DGDG was higher than that of Linseed TAG and Akamoku MGDG (Table 2 and Figure 3).

Overall, the results for the oxidative stability of GLs and TAG obtained in the present study were very different from those expected from the average number of bis-allylic positions and fatty acid composition of each lipid. Our results showed that GL was oxidatively more stable than the TAG form. Furthermore, compared with different types of GLs, DGDG showed a higher oxidative stability than MGDG.

A study by Lee et al. [22] showed that Spinach GL contained a low ratio of saturated fatty acid to unsaturated fatty acid, but the GL had greater oxidative stability than did lipids of other classes such as neutral lipid and phospholipids. We also reported a higher oxidative stability for GL than TAG, although the average number of bis-allylic positions of GL was much higher than that of the TAG [4]. These results indicate that GL has unique characteristics and that the mechanisms involved in oxidation of this class of lipids are unique.

Minor contaminant compounds in the GL prepared from photosynthetic organisms such as plant and seaweeds are known to affect the oxidative stability of GL. Chlorophyll, a representative impurity, has been reported to have both antioxidant [23] [24] and pro-oxidant [25] activities. However, chlorophyll was removed successfully from the samples by double purification, first using silicic acid and then a carbon column as described in the experimental procedures. Additionally, experiments were conducted in the dark, minimizing the opportunity for traces of chlorophyll to react with light. Carotenoids and tocopherols may also have contaminated the GL. These antioxidants were also completely removed from the purified GL used in the present study. Furthermore, Linseed TAG was completely free from antioxidants and pro-oxidants such as oxidation products.

PUFA oxidation essentially proceeds via free radical chain reaction, where the rate-limiting step is the abstraction of $\mathrm{H} \bullet$ from the bis-allylic positions of the substrate, PUFA. Therefore, the susceptibility of PUFA to oxidation depends on the availability of bis-allylic hydrogens. In contrast, physical and stereochemical protection of bis-allylic hydrogens by other molecules has been also regarded as an important factor for understanding the oxidative stability of PUFA, particularly in oil in water emulsion, food, and biological systems [26].

In food emulsions, various molecules are distributed according to their polarity and surface activity between different phases, which include the oil phase, water phase, and interfacial region. Lipid oxidation in this system is an interfacial phenomenon that is greatly influenced by the nature of the interface. In this case, emulsifiers greatly affect the oxidative stability of PUFA. At the droplet interface, emulsifier molecules arrange themselves in an interface with PUFA and water so that the polar head-groups are located at the surface and non-polar tails are in the interior; bis-allylic positions of PUFA can be protected by the interactions with emulsifiers [26]. 
In phosphatidylcholine liposomes, the model system of biological membranes, DHA showed higher oxidative stability than LA [27] [28]. These results can be explained by the protective conformation of the bis-allylic positions of DHA against hydrogen abstraction. The characteristic structure of the DHA (22:6n-3) moiety has been observed in several membrane models. A molecular modeling approach showed that DHA of diacylglycerol may uniquely influence acyl chain packing arrangements in cell membranes [29] [30] [31]. When in these conformations, DHA hexanes can form tightly packed arrangements to protect the bis-allylic positions of DHA from oxidative attack.

Therefore, the characteristic oxidative stability of GL found in the present study did not result from contamination, but rather from differences in the chemical structure of each lipid molecule. MGDG and DGDG contain both polar (galactosyl moiety) and non-polar (fatty acyl group) regions on the same molecule, which can form a self-assembly structure [32]. In this structure, galactosyl moieties may protect the bis-allylic positions of PUFA in GL through various interactions. The present study revealed the higher oxidative stability of DGDG than that of MGDG for the first time. This may be because of the stronger protective effect of di-galactosyl moieties on PUFA against oxidative attack than that of mono-galactosyl moieties.

\section{Conclusion}

In the present study, we found that PUFAs in the form of GLs were oxidatively more stable than those of TAG. In addition, when the oxidative stability of MGDG and DGDG from Spinach and Akamoku was compared, DGDG showed higher oxidative stability than its corresponding MGDG. These results improve the understanding of the oxidation of GL containing PUFA in biological systems and may be useful for preventing PUFA from undergoing oxidative deterioration. Further studies are required to elucidate the detailed mechanism of the unusually high oxidative stability of GLs.

\section{Acknowledgements}

This work was supported by the "Science and technology research promotion program for agriculture, forestry, fisheries and food industry" from the Ministry of Agriculture, Forestry and Fisheries in Japan. The author, Noor Yuslida binti Hazahari, would like to express her sincere thanks and gratitude to the Ministry of Education, Culture, Sports, Science and Technology (MEXT, Japan) for supporting her study in Hokkaido University, Hakodate Campus.

\section{References}

[1] Harwood, J.L. (1980) Plant Acyl Lipids: Structure, Distribution and Analysis. In: Stump, P.K. and Conn, E.E., Eds., Biochemistry of Plants, Vol. 4, Academic Press, New York, 1-55. https://doi.org/10.1016/B978-0-12-675404-9.50007-2

[2] Harwood, J.L. and Jones, A.L. (1989) Lipid Metabolism in Algae. Advances in Botanical Research, 16, 1-53. https://doi.org/10.1016/S0065-2296(08)60238-4 
[3] Thompson Jr., G.A. (1996) Lipids and Membrane Function in Green Algae. Biochimica et Biophysica Acta, 1302, 17-45. https://doi.org/10.1016/0005-2760(96)00045-8

[4] Yamaguchi, T., Sugimura, R., Shimajiri, J., Suda, M., Abe, M., Hosokawa, M. and Miyashita, K. (2012) Oxidative Stability of Glyceroglycolipids Containing Polyunsaturated Fatty Acids. Journal of Oleo Science, 61, 505-513.

[5] Dörmann, P. and Benning, C. (2002) Galactolipids Rule in Seed Plants. Trends in Plant Sciences, 7, 112-118. https://doi.org/10.1016/S1360-1385(01)02216-6

[6] Terasaki, M., Hirose, A., Narayan, B., Baba, Y., Kawagoe, C., Yasui, H., Saga, N., Hosokawa, M. and Miyashita, K. (2009) Evaluation of Recoverable Functional Lipid Components with Special Reference to Fucoxanthin and Fucosterol Contents of Several Brown Seaweeds of Japan. Journal of Phycology, 45, 974-980.

https://doi.org/10.1111/j.1529-8817.2009.00706.x

[7] Miyashita, K. and Takagi, T. (1986) Study on the Oxidative Rate and Prooxidant Activity of Free Fatty Acids. Journal of the American Oil Chemists' Society, 63, 1380-1384. https://doi.org/10.1007/BF02679607

[8] Cosgrove, J.P., Church, D.F. and Pryor, W.A. (1987) The Kinetics of the Autoxidation of Polyunsaturated Fatty Acids. Lipids, 22, 299-304. https://doi.org/10.1007/BF02533996

[9] Cho, S.-Y., Miyashita, K., Miyazawa, T., Fujimoto, K. and Kaneda, T. (1987) Autoxidation of Ethyl Eicosapentaenoate and Docosahexaenoate. Journal of the American Oil Chemists' Society, 64, 876-879. https://doi.org/10.1007/BF02641498

[10] Meloni, D.A., Oliva, M.A., Martinez, C.A. and Cambraia, J. (2003) Photosynthesis and Activity of Superoxide Dismutase, Peroxidase and Glutathione Reductase in Cotton under Salt Stress. Environmental and Experimental Botany, 49, 69-79. https://doi.org/10.1016/S0098-8472(02)00058-8

[11] Nunez, M., Mazzafera, P., Mazorra, L.M., Siquira, W.J. and Zullo, M.A.T. (2003) Influence of a Brassinosteroid Analogue on Antioxidant Enzymes in Rice Grown in Culture Medium with NaCl. Biologia Plantarum, 47, 67-70. https://doi.org/10.1023/A:1027380831429

[12] Yasar, F., Ellialtioglu, S. and Yildiz, K. (2008) Effect of Salt Stress on Antioxidant Defense Systems, Lipid Peroxidation, and Chlorophyll Content in Green Bean. Russian Journal of Plant Physiology, 55, 782-786. https://doi.org/10.1134/S1021443708060071

[13] Kuroe, M., Kamogawa, H., Hosokawa, M. and Miyashita, K. (2016) Dietary Spinach ALA Enhances Liver n-3 Fatty Acid Content to Greater Extent than Linseed Oil in Mice Fed Equivalent Amounts of ALA. Lipids, 51, 39-48. https://doi.org/10.1007/s11745-015-4086-9

[14] Yang, Y.-H., Du, L., Hosokawa, M., Miyashita, K., Kokubun, Y., Arai, H. and Taroda, H. (2017) Fatty Acid and Lipid Class Composition of the Microalga Phaeodactylum tricornutum. Journal of Oleo Sciences, 66, 363-368.

[15] Uemura, M., Shibata, A., Hosokawa, M., Iwashima-Suzuki, A., Shiota, M. and Miyashita, K. (2016) Inhibitory Effect of Dihydrosphingosine with $\alpha$-Tocopherol on Volatile Formation during the Autoxidation of Polyunsaturated Triacylglycerols. Journal of Oleo Science, 65, 713-722.

[16] Prevot, A.F. and Modret, F.X. (1976) Utilisation des Colonnes Capillaries de Verre Pour l'Analyse des Corps Gras Par Chromatographie en Phase Gazeuse. Revue Française des Corps Gras, 23, 409-423.

[17] Shimajiri, J., Shiota, M., Hosokawa, M. and Miyashita, K. (2013) Synergistic Anti- 
oxidant Activity of Milk Sphingomyeline and Its Sphingoid Base with $\alpha$-Tocopherol on Fish Oil Triacylglycerol. Journal of Agricultural and Food Chemistry, 61, 7969-7975. https://doi.org/10.1021/jf401788j

[18] Miyashita, K. (2008) Oxidation of Long-chain Fatty Acids. In: Kamal-Eldin, A. and Min, D., Eds., Lipid Oxidation Pathways, AOCS Press, Champaign, IL, 54-78.

[19] Frankel, E.N. (1998) Free Radical Oxidation, Hydroperoxide Formation. In: Frankel, E.N., Ed., Lipid Oxidation, The Oily Press, Dundee, Scotland, 13-41.

[20] Porter, N.A., Caldwell, S.E. and Mills, K.A. (1995) Mechanisms of Free Radical Oxidation of Unsaturated Lipids. Lipids, 30, 277-290.

https://doi.org/10.1007/BF02536034

[21] Miyashita, K., Frankel, E.N., Neff, W.E. and Awl, R.A. (1990) Autoxidation of Polyunsaturated Triacylglycerols. III. Syntheytic Triacylglycerols Containing Linoleate and Linolenate. Lipids, 25, 48-53. https://doi.org/10.1007/BF02562427

[22] Lee, J., Park, K., Lee, S., and Choe, E. (2000) Lipid Changes of Freeze-Dried Spinach by Various Kinds of Oxidation. Journal of Food Science, 65, 1290-1295. https://doi.org/10.1111/j.1365-2621.2000.tb10599.x

[23] Endo, Y., Usuki, R. and Kaneda, T. (1985) Antioxidant Effects of Chlorophyll and Pheophytin on the Autoxidation of Oils in the Dark II. The Mechanism of Antioxidant Action of Chlorophyll. Journal of the American Oil Chemists' Society, 62, 839-1841.

[24] Endo, Y., Usuki, R. and Kaneda, T. (1985) Antioxidant Effects of Chlorophyll and Pheophytin on the Autoxidation of Oils in the Dark I. Comparison of the Inhibitory Effects. Journal of the American Oil Chemists' Society, 62, 1375-1378. https://doi.org/10.1007/BF02545962

[25] Frankel, E.N. (1998) Photooxidation of Unsaturated Fats. In: Frankel, E.N., Ed., Lipid Oxidation, The Oily Press, Dundee, Scotland, 43-54.

[26] Miyashita, K. (2014) Paradox of Omega-3 PUFA Oxidation. European Journal of Lipid Science and Technology, 116, 1268-1279. https://doi.org/10.1002/ejlt.201400114

[27] Nara, E., Miyashita, K. and Ota, T. (1997) Oxidative Stability of Liposomes Prepared from Soybean PC, Chicken Egg PC, and Salmon Egg PC. Bioscience, Biotechnology, and Biochemistry, 61, 1736-1738. https://doi.org/10.1271/bbb.61.1736

[28] Araseki, M., Yamamoto, K. and Miyashita, K. (2002) Oxidative Stability of Polyunsaturated Fatty Acid in Phosphatidylcholine Liposomes. Bioscience, Biotechnology, and Biochemistry, 66, 2573-2577. https://doi.org/10.1271/bbb.66.2573

[29] Applegate, K.R. and Glomset, J.A. (1986) Computer-Based Modeling of the Conformation and Packing Properties of Docosahexaenoic Acid. Journal of Lipid Research, 27, 658-680.

[30] Applegate, K.R. and Glomset, J.A. (1991) Effect of Acyl Chain Unsaturation on the Conformation of Model Diacylglycerols: A Computer Modeling Study. Journal of Lipid Research, 32, 1635-1644.

[31] Applegate, K.R. and Glomset, J.A. (1991) Effect of Acyl Chain Unsaturation on the Packing of Model Diacylglycerols in Simulated Monolayers. Journal of Lipid Research, 32, 1645-1655.

[32] Chaiyasit, W., Elias, R.J., McClements, D.J. and Decker, E.A. (2007) Role of Physical Structure in Bulk Oils on Lipid Oxidation. Critical Reviews in Food Science and Nutrition, 47, 299-317. https://doi.org/10.1080/10408390600754248 